https://www.journal-imab-bg.org

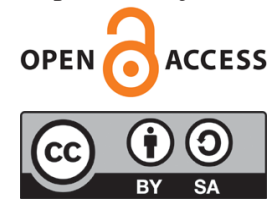

Original article

\title{
SCIENTIFIC EXERCISES APPROACH TO SCOLIOSIS (SEAS) METHODOLOGY IN ADOLESCENT IDIOPATHIC SCOLIOSIS
}

\author{
Stamenka Mitova \\ Training sector of Kinesitherapy, Faculty of Public Health, Healthcare and \\ Sports, Department of Kinesitherapy, South-West University Neofit Rilski, \\ Blagoevgrad, Bulgaria.
}

\section{SUMMARY}

The aim of this study is to investigate the changes in the spine, post-training in corrective exercises with the SEAS method in moderate idiopathic scoliosis.

Material and Methods: A contingent in the present study was 42 subjects with mild adolescent idiopathic scoliosis. The methodology of research includes conducting functional studies and tests - an anamnesis (including physical exercise data, motor behavior, etc.), somatoscopy (examination), radiographic and postural assessment, GPS100 system test, and Adams test. Physiotherapy methodology includes training in specific corrective exercises for scoliosis by the SEAS method with a duration of 7 days of 60 minutes.

Results: 42 subjects with mild adolescent idiopathic scoliosis were studied, of which 26 girls and 16 boys with a follow-up time of 12 months. Mean values of height and weight are as follows for girls $156.3 \pm 7.26 \mathrm{~cm}$ and 49.23 $\pm 4.59 \mathrm{~kg}$, for boys $158.1 \pm 5.27 \mathrm{~cm}$ and $52.88 \pm 4.76 \mathrm{~kg}$. The mean Cobb's angle of scoliosis in girls was $20.08^{\circ} \pm 2.47^{\circ}$, for boys $23.06^{\circ} \pm 3.66^{\circ}$.

Changes in Cobb's angle in girls and boys are reported. The mean Cobb's angle in pre-treatment girls from $20.08^{\circ} \pm 2.47^{\circ}$ to $15.73^{\circ} \pm 2.05^{\circ}$ at the end of the follow-up $(\mathrm{p}<0.001)$. In pretreatment boys at $23.06^{\circ} \pm 3.66^{\circ} \mathrm{Cobb}$, after 12 months of correction exercise the SEAS angle was $17.25^{\circ} \pm 2.96^{\circ},(\mathrm{p}<0.001)$. The most significant influence is in the boys.

Conclusions: SEAS is an approach to the treatment of scoliosis, which differs from many others due to continuous improvement and development, to maintain the evidence base through research results.

Keywords: SEAS, spinal column, research, prevention, treatment

\section{INTRODUCTION}

Idiopathic scoliosis is one of the most common deformities of the vertebral column, unknown etiology. According to different theories, the causes may be biomechanical, genetic, neuromuscular, neurogenic, but the understanding of the scoliosis etiology is still limited. Determining the cause is crucial for effective treatment [1].
Idiopathic scoliosis is a three-dimensional deformation of the growing vertebral column, affecting $2 \%-3 \%$ of adolescents. Scoliosis is classified according to whether there is a specific cause. The disease can lead to significant impairment of body morphology, reduced chest volume, respiratory distress, increased frequency, back pain and serious aesthetic problems. The risk of deterioration is highest during puberty and increases the risk of pathological spinal cord injury if the extreme curvature exceeds a certain critical threshold, the risk of health problems and the progression of the curve increases. Early clinical discovery of scoliosis is based on careful examination of the spine and is the subject of screening programs. The treatment options are physiotherapy, corrective strengthening or surgery [2-7].

Observations, specific physiotherapeutic exercises for scoliosis and growth during growth are all therapeutic interventions adopted by the International Society of Orthopedic and Rehabilitation Scales (SOSORT). The standard features of these interventions are: 1) 3-dimensional self-correction; 2) day-to-day activities and; 3) stabilizing the corrected posture.

Standing and keeping, their maintenance and care depend on the correct shape of the spine, on the proper functioning of the musculature, on its strength, on the even distribution of the muscle thrust, that is to say on the harmonic work of all the muscles involved in the movements of the spine $[8,9]$.

SEAS is an abbreviation of the „Scientific Exercise Approach to Scoliosis“, which is linked to ongoing changes in the research-based approach. SEAS is a method of treating scoliosis created on a modern neurophysiological basis and based on the SOSORT rods [10].

SEAS is a method of treating scoliosis that focuses on restoring postural control and improving spine stability through exercises involving active 3D self-correction of the scoliotic posture. The active 3D correction is accomplished by training the patient, that is, "adapted" to the patient's ability (active self-correction) within the 3D spatial planes. The SEAS method then focuses on spinal column stabilization and posture maintenance through various specialized exercises to achieve sub-conscious self-correction of posture by stimulating neurosensory posture mechanisms. 
The SEAS works with a specific difference with bracing: in fact, while an orthotic device can change continuously the posture of the patient making it somehow fixed exercises can only determine behaviouraland automatic changes of movement and posture through different motor control strategies. This is particularly important for a bodily system like the trunk and spine, that has been demonstrated to be driven more by automatic, feed-forward schemes than voluntary control. Moreover, as shown by Stokes, active movement is more effective than passive positioning in determining changes of spinal deformity: this is one of the reasons for an active exercises approach as a stand-alone treatment or coupled with bracing according to the so-called active bracing principle. In this theoretical framework, SEAS exercises are based on autocorrection and stabilisation $[10,11,12,13]$.

\section{MATERIAL AND METHODS}

A contingent in the present study is 42 subjects with mild adolescent idiopathic scoliosis, of which 26 are girls and 16 are boys. The study was conducted for 12 months (January 2018 - January 2019) at the Bachinovo Sports Research and Recreation Center, South-West University „Neofit Rilski“ in Blagoevgrad, after signing the informed consent statements.

The mean age of the observed contingent $(\bar{X} \pm \mathrm{SD})$ in girls was $11.15 \pm 0.78$ yearsand in boys $11.25 \pm 0.86$ years. The study methodology includes conducting functional studies and tests - an anamnesis (including physical exercise data, motor behavior, etc.), somatoscopy (examination), radiographic and postural evaluation, GPS100 study, Adams test.

The methodology of physiotherapy involves training in specific corrective exercises for scoliosis by the SEAS method, lasting 7 days 60 minutes. Patients become acquainted with their scoliotic deformation and corpse changes. The sequence of corrective movements is selected according to patient pathology and morphological peculiarities. They are active and self-sustaining. Stabilization is achieved with isomeric contraction. After training, patients receive a complete program with specific home improvement exercises within 20-30 minutes, at least 3 times a week. Once a week, individual classes are held.

The main purpose of this study is to track the changes in the spine after training in SEAS correction exercises in adolescent idiopathic scoliosis.

The main tasks are: 1) training in specific physiotherapeutic exercises focused on restoring postural control and improving the stability of the spine through exercises involving active 3D self-correction of the scoliotic posture; 2) Building and consolidating a self-correction habit; 3) spinal column stabilization and posture maintenance through various specialized exercises, to achieve post-self-regulation by stimulating neurosensory mechanisms to maintain posture; 4) Positive psycho-emotional impact and motivation of the child actively and willingly to participate in the activities; 5) restoring mus- cle balance, increasing muscle strength; (6) improving the function of the respiratory system, training in proper breathing, and improving static and dynamic control of the spine.

The treatment of adolescent idiopathic scoliosis is mildly not operative and includes a multidisciplinary team of doctor, orthopedic surgeon, physiotherapist, psychologist and family.

\section{RESULTS}

42 subjects with mild adolescent idiopathic scoliosis were studied, of which 26 girls and 16 boys with a follow-up time of 12 months. Mean values of height and weight are as follows for girls $156.3 \pm 7.26 \mathrm{~cm}$ and $49.23 \pm 4.59 \mathrm{~kg}$, for boys $158.1 \pm 5.27 \mathrm{~cm}$ and $52.88 \pm 4.76 \mathrm{~kg}$. The mean angle of scoliosis in girls was $20.08^{\circ} \pm 2.47^{\circ}$, for boys $23.06^{\circ} \pm 3.66^{\circ}$ for Cobb.

Changes in Cobb's angle in girls and boys are reported. The average Cobb's angle in pre-therapy girls from $20.08^{\circ} \pm 2.47^{\circ}$ to $15.73^{\circ} \pm 2.05^{\circ}$ at the end of the follow-up $(\mathrm{p}<0.001)$. In boys before therapy $23.06^{\circ} \pm 3.66^{\circ}$ Cobb's angle, after 12 months of correction exercise, the SEAS angle was $17.25^{\circ} \pm 2.96^{\circ}(\mathrm{p}<0.001)$. The most significant influence is in the boys.

\section{DISCUSSION}

Our results indicate that there is a reduction in the Cobb's angle in the investigated contingent after specific SEAS correction exercises for scoliosis within 12 months. Our results are similar to those of 21 children aged 13.6 \pm 1.4 years with idiopathic scoliosis, with Schroth's training under the Barcelona School Scoliosis Physical Therapy (BSPTS) system. The cases are divided into three groups according to the angle of Cobb - light to medium scoliosis $\left(15^{\circ}-24^{\circ}\right)$, medium $\left(25^{\circ}-34^{\circ}\right)$ and medium to heavy scoliosis $\left(35^{\circ}-44^{\circ}\right)$. The mean follow-up is 6 months with proven safety for the treatment. Changes in the Cobb angle, torso rotation angle and POTSI rear symmetry index were reported. At the end of the 13-month follow-up, $48 \%$ improvement and $33 \%$ stabilization of scoliosis were reported. At $19 \%$, the curvature has progressed despite therapy. The most significant improvement is seen in the mid to severe scoliosis group, with an average Cobb angle reduction of $37.4^{\circ} \pm 2.7^{\circ}$ before the treatment to $28.1^{\circ} \pm 2.4^{\circ}$ $(\mathrm{p}<0.001)$ in the end of tracking [14].

\section{CONCLUSION}

SEAS are an approach to the treatment of scoliosis, which differs from many others due to continuous improvement and development, to maintain the evidence base through research results.

\section{Acknowledgements:}

This article is supported under Project No. RP-A1/ 19, Project topic: Research the possibilities of laser acupuncture for musculoskeletal dysfunctions in the spinal column region. 


\section{REFERENCES:}

1. Fadzan M, Bettany-Saltikov J. Etiological Theories of Adolescent Idiopathic Scoliosis: Past and Present. Open Orthop J. 2017 Dec 29;11:14661489. [PubMed] [Crossref]

2. Kotwicki T, Chowanska J, Kinel E, Czaprowski D, Tomaszewski M, Janusz P. Optimal management of idiopathic scoliosis in adolescence. Adolesc Health Med Ter. 2013 Jul 23; 4:59-73. [PubMed] [Crossref]

3. Hresko MT. Clinical practice. Idiopathic scoliosis in adolescents. $N$ Engl $J$ Med 2013; 368:834-41. [PubMed]

4. Dickson RA, Lawton JO, Archer IA, Butt WP. The pathogenesis of idiopathic scoliosis. Biplanar spinal asymmetry. J Bone Joint Surg Br. 1984; 66:8-15. [PubMed]

5. Bettany-Saltikov J, Weiss HR, Chockalingam N, Taranu R, Srinivas S, Hogg J, et al. Surgical versus non-surgical interventions in people with adolescent idiopathic scoliosis. Cochrane Database Syst Rev. 2015 Apr 24;(4):CD010663 [PubMed]

6. Chongov B, Alexiev V, Georgiev H, Kalinov K, Dimitrova E. Correlation between scoliosis deformity type and trunk symmetry before and after schroth physiotherapeutic exercises. $C$ $R$ Acad Bulg Sci. 2017 Nov;70(10): 1455-1462. [Internet]

7. Gramatikova M. Aquatherapy after reconstruction of the anterior cruciate ligament of knee joint. Activities in Physical Education and Sport 2015, International Journal of Scientific and Professional Issues in Physical Education and Sport. 5(1):33-36.

8. Mitova S, Popova D, Gramatikova M. Frequency and prevalence of postural disorders and spinal deformities in children of primary school age. Research in Kinesiology 2015; 43(1): 21-24.

9. Gramatikova M. Kinesiotherapy for dynamic knee joint instability, textbook of a monographic nature, University Publishing House SWU „Neofit Rilski”, Blagoevgrad, 2017. 200 p. [in Bulgarian]

10. Romano M, Negrini A, Parzini S, Tavernaro M, Zaina F, Donzelli S, et al. SEAS (Scientific Exercises Approach to Scoliosis): a modern and effective evidence based approach to physiotherapic specific scoliosis exercises. Scoliosis. 2015 Feb 5;10:3. [PubMed] [Crossref]

11. Zaina F, De Mauroy JC, Grivas T, Hresko MT, Kotwizki T, Maruyama $\mathrm{T}$, et al. Bracing for scoliosis in 2014: state of the art. Eur J Phys Rehabil Med. 2014 Feb;50(1):93-110. [PubMed]

12. Romano M, Minozzi S, Zaina F, Saltikov JB, Chockalingam N, Kotwicki T, et al. Exercises for adolescent idiopathic scoliosis: a Cochrane systematic review. Spine (Phila $\mathrm{Pa}$ 1976). 2013 Jun 15;38(14):E883-93. [PubMed]

13. Bettany-Saltikov J, Parent E, Romano M, Villagrasa M, Negrini S. Physiotherapeutic scoliosis-specific exercises for adolescents with idiopathic scoliosis. Eur J Phys Rehabil Med. 2014 Feb;50(1):111-21. [PubMed]

14. Chongov B, Alexiev V, Georgiev H, Kalinov K, Dimitrova E. Correlation between scoliosis deformity type and trunk symmetry before and after schroth physiotherapeutic exercises. C R Acad Bulg Sci. 2017 Nov;70(10):1455-1462. [Internet]

Please cite this article as: Mitova S. Scientific Exercises Approach to Scoliosis (SEAS) Methodology in Adolescent Idiopathic Scoliosis. J of IMAB. 2020 Jul-Sep;26(3):3333-3335. DOI: https://doi.org/10.5272/jimab.2020263.3333

Received: 24/09/2019; Published online: 23/09/2020

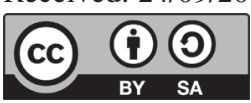

Address for correspondence:

Assoc. Prof. Stamenka Mitova, PhD

Faculty of Public Health, Healthcare and Sports

Department of Kinesitherapy, South-West University Neofit Rilski

66, Ivan MihaylovStr., 2700 Blagoevgrad, Bulgaria.

E-mail: stami80@abv.bg 